FACTA UNIVERSITATIS

Series: Automatic Control and Robotics Vol. 20, Nº 1, 2021, pp. 33 - 42

https://doi.org/10.22190/FUACR201231003A

Regular Paper

\title{
ARTIFICIAL NEURAL NETWORKS APPLICATION TO PREDICTION OF ELECTRICITY CONSUMPTION
}

$U D C(621.317 .38: 004.032 .26)$

\section{Miona Andrejević Stošović, Novak Radivojević, Igor Jovanović, Andrija Petrušić}

\begin{abstract}
University of Niš, Faculty of Electronic Engineering, Department of Electronics, Republic of Serbia

Abstract. In this paper, we will present an artificial neural network (ANN) model trained to forecast hourly electricity consumption of energy in industry for a day-ahead. We will start with a brief analysis of the global electricity market with a special reference to the Serbian market. Next, the daily electricity consumption amounts between August $1^{\text {st }}$ and December $19^{\text {th }} 2019$ will be analyzed using statistical tools. According to the obtained results, we will give predictions of our models, based on different number of previous days.
\end{abstract}

Key words: ANN, energy consumption, forecasting, seasonality

\section{INTRODUCTION}

Liberalization of electric power sector in the last two decades led to very dynamic wholesale markets and deregulated retail markets. At wholesale market level, traders are focused on price dynamics trends, while on retail level for supplying electricity to final consumers the consumption forecast can make all the difference for fostering costefficiency [1].

Furthermore, forecasting energy consumption on short-term, mid-term and long-term level is essential when defining strategies for production planning and expansion of infrastructure capacities for electric power systems.

A good approach to consumption prediction of individual consumers can have a farreaching effect on the aggregated level. Strategic decision-making tools are more reliable when based on proper models for determining patterns of individual consumptions for various types of industries.

Received December 31, 2020 / Accepted March 19, 2021

Corresponding author: Miona Andrejević Stošović

University of Niš, Faculty of Electronic Engineering, Aleksandra Medvedeva 14, 18000 Niš, Republic of Serbia

E-mail: miona.andrejevic@elfak.ni.ac.rs 
It can be observed on the example of electric power suppliers as balance responsible parties at liberalized energy markets how important is having an adequate mathematical model for forecasting individual consumption. The balance responsibility refers to the penalty-based financial mechanism developed to force market participants to properly anticipate their energy needs on a short-term level.

An electric power supplier is obliged to inform, in a day-ahead manner, a transmission system operator (TSO) how much of electric power needs to be provided on hourly level for all his electricity consumers. This is important, because TSO needs to maintain the balance in real-time between production and consumption for entire energy system, based on the data provided from all balancing parties active on the market. There is a certain tolerance that TSO will allow to balancing parties, but at the end if anticipated hour consumptions are not matching actual consumed energy and the tolerance for imbalance goes above established limits, the balancing party will be penalized through settlement prices defined for each hour retroactively based on pre-established methodology issued by Regulatory body.

In liberalized power markets, balance responsibility and imbalance settlement are two closely related elements that constitute the essential part of a balancing market, which is an institutional arrangement establishing market-based balancing [2].

In the end, market participant, proportionally to the imbalance they created in the system for each hour, pays generated imbalance costs. If an Electric power supplier lacks adequate forecasting tool and gives poor judgment on how much electricity is needed in each hour, and for each day, for all its consumers that are in his balancing group, unnecessary expense occurs for each estimation that at the end is above the allowed imbalance limits for measured consumption.

If consumption of the balancing group is observed only on an aggregated level, many studies have shown that there is a correlation with outside temperature, but balancing group dynamics is mostly out of focus. Therefore, the smaller balancing group is (fewer metering points and less volume), considering the fluctuation of clients is on yearly level, there is more need for Electric power supplier to estimate balancing group needs through forecasting models of individual energy consumptions for each metering point.

The importance of electricity consumption forecasting on one hand, and its complexity on the other hand, have motivated many researchers in this area. In the literature, there are numerous studies on electricity consumption and demand estimation. In these studies, some of commonly used methods are stationary time series models [3], regression models [4], [5], [6], econometric models [7]. However, most of time series models are linear predictors, while electricity consumption is inherently a nonlinear function. So, the behavior of electricity consumption series may not be completely captured by the time series techniques. To solve this problem, some other research papers have proposed Artificial Neural Networks (ANN) and genetic algorithms for electricity consumption forecasting [8], [9].

The main purpose of this study using measured hourly-based consumption data is to develop an adequate forecasting model for predicting electricity consumption for individual consumers that can be used as a proper tool by an Electric Power Supplier to avoid unnecessary imbalance costs to properly estimate energy needs on an hourly and daily level.

For the purpose of this study, United Green Energy Ltd. [10] consulting company for Energy market has provided data of an individual consumer. Data was strategically chosen so irregular intermittent consumption profile can be used as the input data set. Furthermore, this consumption profile lacks correlation with global factors (e.g. outside temperature), as it is mostly dependable on the business potential company that creates for itself. 
Collected hourly data is presented in the form of the time series for the period August $1^{\text {st }}-$ December $19^{\text {th }} 2019$.

\section{TIME-SERIES}

When observing the change in the value of a quantity over time, it is convenient to present these values in the form of time-series. A time-series is a series of data arranged in chronological order. The data in that sequence represents the value of a quantity at discrete moments of time, by which the trend of a given quantity can be determined - at what intervals it increases or decreases, when it has a maximum or minimum value, whether the values of a given quantity change according to a pattern, etc. In Fig.1. an example of a time series representing the power consumption of a company in the period from August $1^{\text {st }}$-December $19^{\text {th }} 2019$ is given. Data were taken every hour and are expressed in kWh.

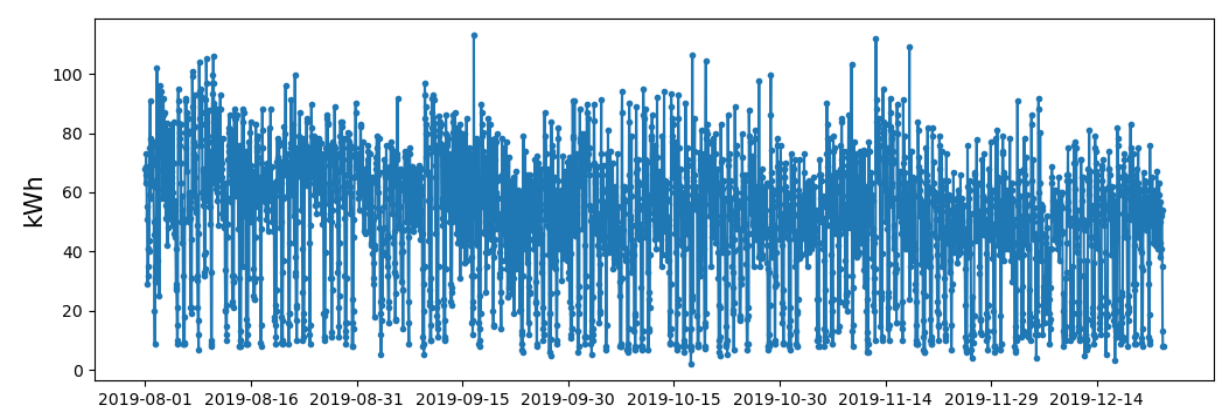

Fig. 1 Time-series of power consumption

Time-series are most often used when it is necessary to solve the problem of predicting the values that a given quantity may take in the future. Problems of predicting the trend of the observed quantity are often encountered in practice, since it is useful to know in advance how a certain quantity will behave. This paper will present an example of one such problem and its solution - for the purposes of planning the cost of consumed power. Analysis of time-series and forecasting is given in [11].

In statistics, there are various mathematical models and methods that can be used to predict a quantity. Usually these methods are based on the assumption that it is possible to predict future values of the observed quantity based on the values it took in previous moments. Various statistical methods and calculations are often used to predict time-series, but in order to be able to apply them, it is usually necessary to know well the nature of the observed time-series. Time-series have several characteristics that describe its shape and behavior, so they can be used to see how predictable a time series is. Stationarity, seasonality and autocorrelation are most often considered characteristics. Sometimes, these characteristics must meet some conditions or otherwise they will not be suitable for analysis, so some additional manipulation of the data set must be performed.

We say that a time-series is stationary if its statistical properties (mean and variance) are constant over time. For statistical analysis, it is convenient for the time series to be stationary, since according to some criteria, its behavior in that case can be easily predicted. Time series are seasonal if there are some seasonal factors that affect its shape, such as time 
of year, or day of the week. In other words, the time series experiences drastic changes at some specific time points, and these changes occur periodically. Seasonality is undesirable because it introduces unpredictability and makes the analysis more complex. Seasonality is described by autocorrelation, that tells us the extent to which a time series is linearly related to its copy being translated by several steps in time. In other words, autocorrelation can determine how two observations, distant from each other, are mutually conditioned. The autocorrelation function for our time series is given in Fig. 2. Statistical methods require many parameter adjustments, data transformations, method upgrades, etc. in order to successfully perform prediction.

Artificial neural networks are often used for this purpose. They are known for being able to be trained to approximate a mathematical function and for some of them are good at recognizing patterns in sequences, which is necessary in this case. There are models of neural networks that predict well even without the need to take into account stationarity and seasonality, because they solve these problems on the fly. In this paper, we will deal with the application of neural network models to power consumption predictions.

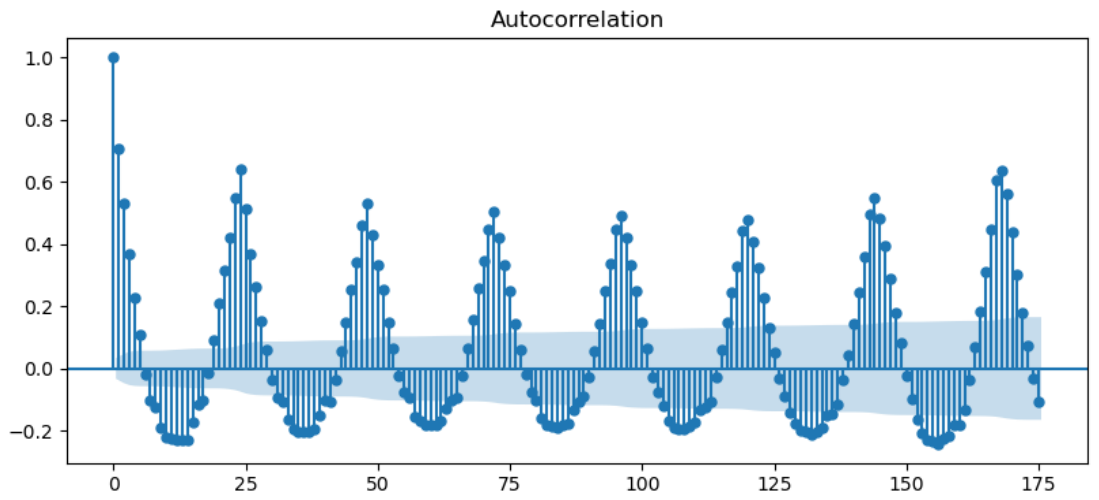

Fig. 2 Autocorrelation function for given time-series

\section{DATA SETUP AND ANN STRUCTURE}

Artificial neural networks have been used for short-time forecasting in electronics [12], [13]. Models were also developed for prediction of electricity consumption on a monthly and weekly level. A similar approach was used in the development of a forecasting tool with the use of ANNs, in order to forecast the energy consumption in the building of Regulation Authority of Energy in Athens city, Greece [14]. Long-term forecasting of electricity energy consumption was presented in [15].

In this paper we will present models based on an artificial neural network for prediction of consumption on an hourly, daily and weekly level. Initially, measured consumption data need to be gathered, managed and prepared for training.

Once the data has been prepared, it is necessary to decide on the length of the input data vector, i.e. what data is needed in order to make a good consumption forecast for the next hour. At the beginning, the length of the input data vector is set to 24 , so the prediction is to be made based on data from the previous 24 hours. Also, the loaded data set needs to be divided into two subsets - training set and test set. The network is trained only using the data from the 
training set, while the test set is used to test the correctness of the predictions - it is not used to train the network but to determine how accurate the network predictions are. Usually the training set makes up $70 \%$ of all data, and the test set takes the rest.

Each training/test set must contain an input vector which has 24 elements and output which is the $25^{\text {th }}$ element and which represents the expected value of the network output. Starting from the first example, each subsequent set is determined by taking data from a time range that is shifted by one hour from that of the previous test set. So, the first training set contains the data from first 24 hours and predicts the $25^{\text {th }}$ hour, the second example contains data from the hour 2 to 25 , and predicts $26^{\text {th }}$, etc.

The network structure used in this paper is a feed-forward Multi-Layer perceptron. It is a fully-connected network consisting of two hidden layers of 32 neurons each. It is previously explained that the network has 24 inputs and one output (predicted value).

This number of hidden neurons was selected after testing models with different numbers of neurons in the hidden layers $(16,32,64,128)$, because for that number the best results were obtained. The ReLU (Rectified Linear Unit) function was used in all nodes as an activation function.

The training process consisted of iterative optimization of network parameters in order to obtain minimal root mean square error between the measured and predicted values, applying the backpropagation algorithm. "Adam" optimization algorithm [16] was used instead of the classical stochastic gradient descent procedure to update network weights iterative based in training data. The network model is described in Python 3.7.9, using the Keras deep learning API from the Tensorflow platform for machine learning [17].

After obtaining a model based on 24 hours input data vector, we can take into account the graph in Fig. 3 for the approximate estimation of the predicted accuracy. Fig. 3 shows a decrease in the value of the error function with an increase in the number of iterations. The total number of iterations is 300 , but the graphs show that for both the training and the test example, the error function converges after the $20^{\text {th }}$ iteration, which means that the network was quickly trained to predict. The mean square errors (MSE) for the entire training and test set were $11.73 \mathrm{kWh}$ and $13.57 \mathrm{kWh}$, respectively.

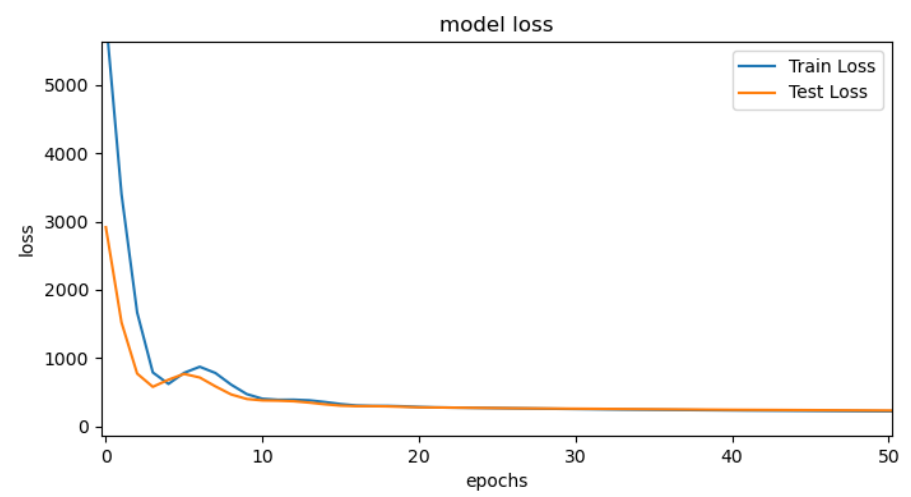

Fig. 3 Prediction error for training set (blue line) and test set (yellow line) as a function of number of iterations

In Fig. 4 the results of the prediction are given. The curve that represents the true value of consumption is blue, and the predicted value is red. Fig. 4a shows the prediction for the 
entire test set, Fig. 4b shows the prediction for one week (taken from the test set), and Fig. $4 \mathrm{c}$ shows prediction for a period of just one day. From the Fig. $4 \mathrm{a}$, we can conclude that ANN predicts very well when consumption is low or medium, but poorly predicts peaks in consumption that exceed $100 \mathrm{kWh}$, because they occur less frequently. In the Fig. 4b we can notice this effect more clearly, and it can also be seen that ANN has created a sense of weekly seasonality, as it predicts a drop in consumption that occurs at the end of the week. Fig. 4c shows a period of approximately 35 hours and it can be seen that there are many spots (hours) in which the network has made accurate predictions and that the model follows the trend well - well predicts an increase and decrease in consumption.

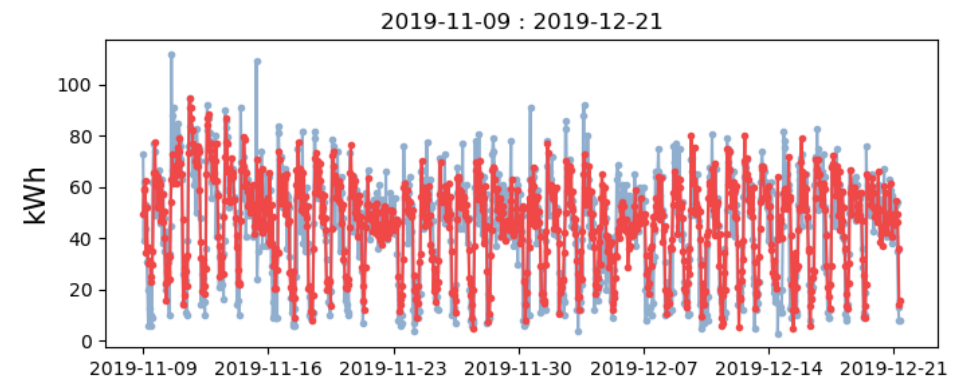

a)

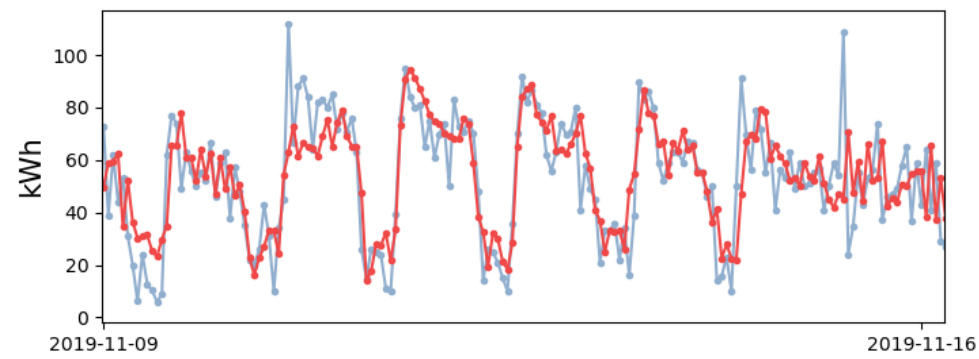

b)

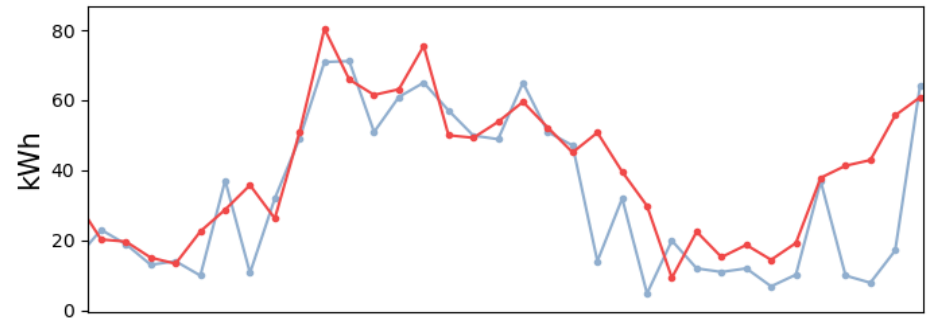

c)

Fig. 4 Prediction results for the entire test set a), one week b), and one day c). (blue lineexpected, red line- predicted)

\section{INFLUENCE OF INPUT VECTOR WIDTH ON FoRECASTING PRECISION}

We will also examine what effect the number of previous measurements (the number of neurons in the input layer of the model) has on the accuracy of the model. In the Fig. 5 
we presented situations when the model predicted with an input vector of $1 \mathrm{~h}, 12 \mathrm{~h}, 24 \mathrm{~h}$, $48 \mathrm{~h}$ and $168 \mathrm{~h}$ (7 days), respectively. The graphs on the left show the forecast on a two-week level, and on the right the forecast of specific cases of sequences - weekend, Monday with one larger peak, and Tuesday without major peaks, can be seen.
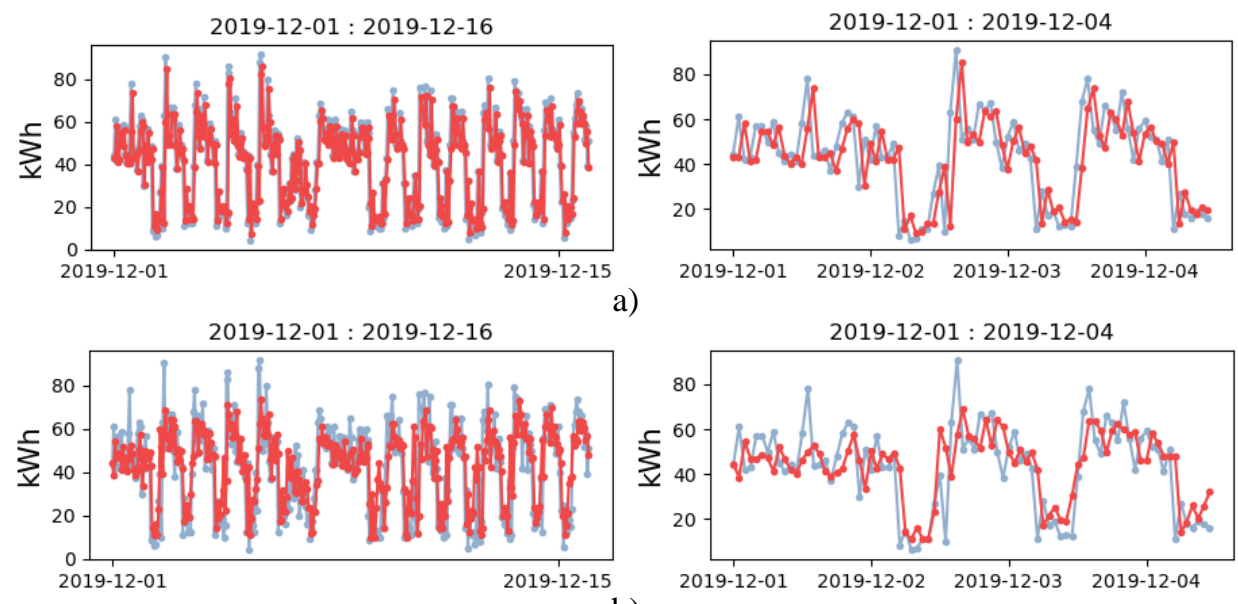

b)
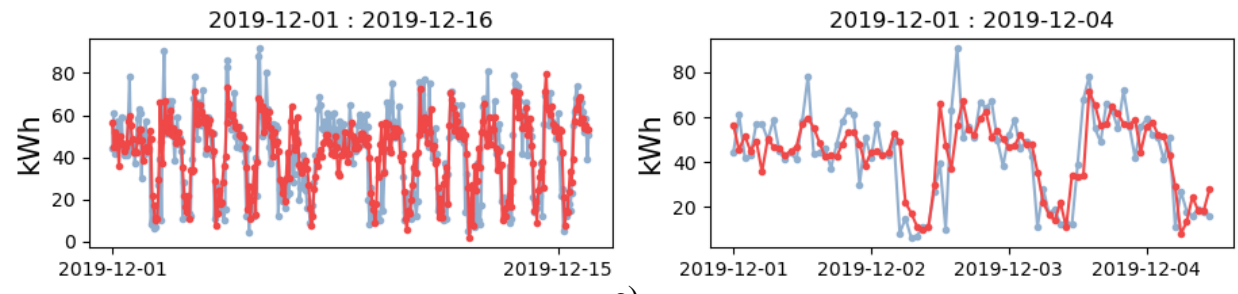

c)
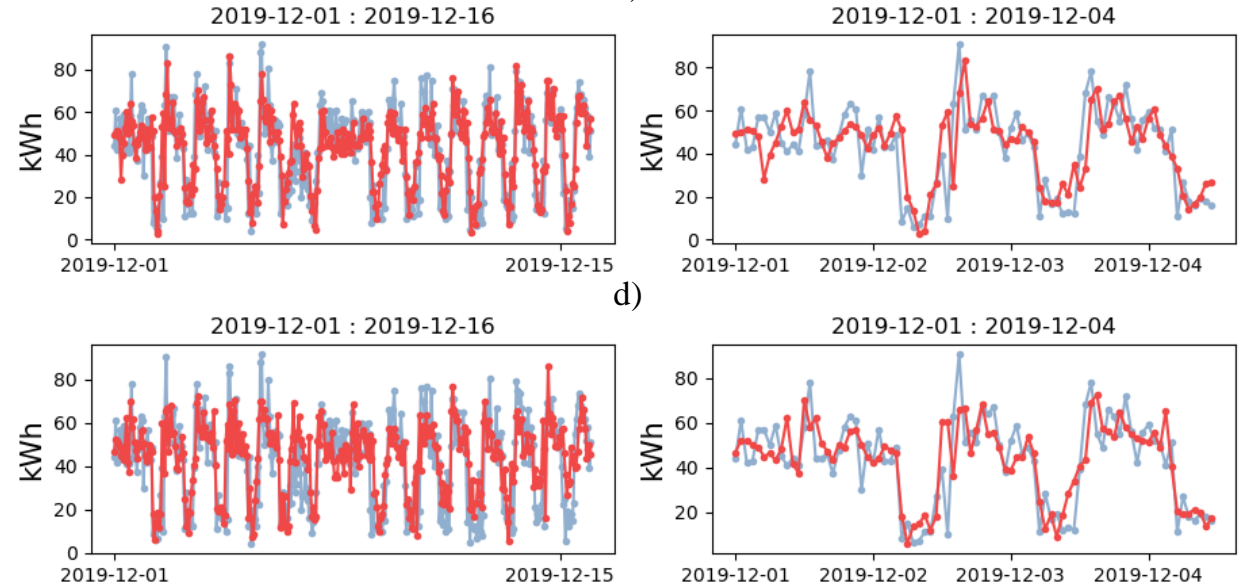

e)

Fig. 5 Predictions for different cases of input data vector width: a) $1 \mathrm{~h}$ shift; b) $12 \mathrm{~h}$ shift; c) $24 \mathrm{~h}$ shift; d) $48 \mathrm{~h}$ shift; e) $168 \mathrm{~h}$ shift; (blue line-expected, red line- predicted) 
Graphs in Fig. 5a show what happens in the extreme case when the model, in the training process, takes into account only the data from the previous hour. At a first glance, it seems that the model predicts very well, but that is not the case at all. Prediction is impossible in this case because the model cannot conclude anything about the consumption trend based on only one data. The model in this case performs the prediction by simply copying the value from the previous step, so the prediction curve appears to have been shifted one hour forward relative to the true consumption curve.

In Figs. 5b, 5c, 5d, it can be seen that the model better predicts peaks, sharper rises and falls as the number of input data increases. The case with 48 input data proved to be optimal in terms of data prediction (Fig 5d), while the case with input data from previous $12 \mathrm{~h}$ best predicts daily oscillations during the weekend (Fig $5 \mathrm{~b}$ ).

Graphs in Fig. 5e represent the case with 168 input data (previous week). This number was chosen following the assumption that the model will learn to predict better if it learns based on data from the entire previous week, because according to the measured data, the same pattern is repeated every week. The model showed an improvement in the prediction for the previous three cases (given in Figs. 5b, 5c, 5d), so it can be assumed that for an even larger data set the prediction will be better. However, it is obvious that the model predicts worse in that case, since it can be seen from the Fig. 5e that the peaks are not covered, and the left graph shows that the model has a drop in consumption during the weekend, which is not the case. This happens due to the overfitting, which is a consequence of a large amount of input data. More input data also reduces the number of training examples, due to the way they are composed. In addition, training examples become more specific as the number of inputs increases- it is less likely that the model will come across an order that is already known to it.

These results can be confirmed if we take into account MSE after training process is completed. Values of these errors for training and test set for all cases are given in the Table 1. The table shows that the model is trained the best in a time step of $168 \mathrm{~h}$, but also that the overfitting effect is present in that case, due to the larger MSE. The case with $48 \mathrm{~h}$ input data has the smallest MSE for the test set, so it can be concluded that this case is optimal.

\begin{tabular}{ccc}
\hline $\begin{array}{c}\text { Table } 1 \text { Mean squared error for training and test se } \\
\text { data }\end{array}$ & $\begin{array}{c}\text { MSE for } \\
\text { training set } \\
{[\mathrm{kWh}]}\end{array}$ & $\begin{array}{c}\text { MSE } \\
\text { for test set } \\
{[\mathrm{kWh}]}\end{array}$ \\
\hline 1 & 16.38 & 15.75 \\
12 & 13.70 & 14.85 \\
24 & 11.73 & 13.57 \\
48 & 11.61 & 13.19 \\
168 & 10.21 & 13.86 \\
\hline
\end{tabular}

\section{FORECASTING ON DAILY LEVEL}

Since information on how much of electric power needs to be provided on hourly level is required a day-ahead, we tried to estimate error of prediction on a daily level. So, prediction for 7 days is done, based on 12, 24, 48 and 168 previous hours. Results are given in Tables II, III, IV, V, respectively. The expected and predicted values in the Tables are obtained as simple summation of values for all hours during one day. From the 
Tables we can see that the greatest errors occur in cases where there are peaks, either with extremely high, or extremely low values. For example, the greatest error is got for the Day 1, in all the Tables. The reason for that is obvious, since the expected value is much less than values for other days, i.e. there is a negative peak. Errors for other days are in the range from $0.8 \%$ to about $9 \%$, what can be considered as quite satisfying.

It is also important to notice that predicted values are in most of the cases greater than expected, since penalty is greater if more power is consumed than it is ordered. These errors have both positive and negative sign, meaning that the total error for a week, as a sum of particular errors, would be much lower.

Table 2 Prediction for 7 days, day by day, as a summation of predictions for all hours based on 12 previous hours

\begin{tabular}{lcrrrrrr}
\hline Day No. & 1 & \multicolumn{1}{c}{2} & \multicolumn{1}{c}{3} & \multicolumn{1}{c}{4} & \multicolumn{1}{c}{5} & \multicolumn{1}{c}{6} & \multicolumn{1}{c}{7} \\
\hline Expected value & 851.2 & 1035.008 & 1155.84 & 1052.16 & 1094.91 & 1243.87 & 1077.24 \\
Predicted value & 974.48 & 1116.08 & 1205.32 & 1101.22 & 1090.49 & 1175.85 & 1056.85 \\
Absolute error & 123.28 & 81.07 & 49.48 & 49.06 & 4.41 & 68.01 & 20.38 \\
Relative error (\%) & -14.48 & -7.83 & -4.28 & -4.66 & 0.40 & 5.46 & 1.89 \\
\hline
\end{tabular}

Table 3 Prediction for 7 days, day by day, as a summation of predictions for all hours based on 24 previous hours

\begin{tabular}{lrlrrrrr}
\hline Day No. & \multicolumn{1}{c}{2} & \multicolumn{1}{c}{3} & \multicolumn{1}{c}{4} & \multicolumn{1}{c}{5} & \multicolumn{1}{c}{6} & \multicolumn{1}{c}{7} \\
\hline Expected value & 851.2 & 1035.008 & 1155.84 & 1052.16 & 1094.91 & 1243.87 & 1077.24 \\
Predicted value & 1040.85 & 1009.69 & 1117.05 & 1103.24 & 1068.58 & 1156.04 & 1119.24 \\
Absolute error & 189.65 & 25.31 & 38.78 & 51.08 & 26.33 & 87.82 & 41.99 \\
Relative error $(\%)$ & -22.28 & 2.44 & 3.35 & -4.85 & 2.4 & 7.06 & -3.89 \\
\hline
\end{tabular}

Table 4 Prediction for 7 days, day by day, as a summation of predictions for all hours based on 48 previous hours

\begin{tabular}{lccrrrrr}
\hline Day No. & 1 & \multicolumn{1}{c}{2} & \multicolumn{1}{c}{3} & \multicolumn{1}{c}{4} & \multicolumn{1}{c}{5} & \multicolumn{1}{c}{6} & \multicolumn{1}{c}{7} \\
\hline Expected value & 851.2 & 1035.008 & 1155.84 & 1052.16 & 1094.91 & 1243.87 & 1077.24 \\
Predicted value & 992.23 & 1099.27 & 1199.04 & 1146.23 & 1077.30 & 1141.84 & 1113.03 \\
Absolute error & 141.03 & 64.27 & 43.20 & 94.07 & 17.60 & 102.03 & 35.78 \\
Relative error $(\%)$ & -16.56 & -6.21 & -3.73 & -8.94 & 1.60 & 8.20 & -3.32 \\
\hline
\end{tabular}

Table 5 Prediction for 7 days, day by day, as a summation of predictions for all hours based on 168 previous hours

\begin{tabular}{lcrrrrrr}
\hline Day No. & \multicolumn{1}{c}{2} & \multicolumn{1}{c}{3} & \multicolumn{1}{c}{4} & \multicolumn{1}{c}{5} & \multicolumn{1}{c}{6} & \multicolumn{1}{c}{7} \\
\hline Expected value & 851.2 & 1035.01 & 1155.84 & 1052.16 & 1094.91 & 1243.87 & 1077.24 \\
Predicted value & 968.14 & 1015.89 & 1112.82 & 1043.13 & 1071.06 & 1141.93 & 1143.67 \\
Absolute error & 116.94 & 19.11 & 43.01 & 9.02 & 23.85 & 101.93 & 66.42 \\
Relative error $(\%)$ & -13.73 & 1.84 & 3.72 & 0.85 & 2.17 & 8.19 & -6.16 \\
\hline
\end{tabular}




\section{CONCLUSION}

The implemented model of the artificial neural network in this example showed satisfactory results in a large number of cases. The model is able to follow the consumption trend, i.e. to predict well when its value increases or decreases, and it is able to adapt to seasonal effects (weekend consumption). The disadvantage of the model is that it often does not well predict a sudden change (increase, decrease) in consumption. It would be desirable for the model to be able to predict consumption peaks as they bring a high cost, so options to improve the performance of the model should be considered. It would be useful to obtain a larger set of consumption data in order to reduce the impact of adjustment and so that the model can better understand the nature of consumption based on new cases. It would be also good to investigate other ways of presenting the time series of consumption and examine its characteristics, e.g. instead of taking data every hour, take it every 15 minutes, or take total consumption for one day instead of every hour.

Acknowledgement: This work has been supported by the Ministry of Education, Science and Technological Development of the Republic of Serbia.

\section{REFERENCES}

[1] Pepermans, G., "European energy market liberalization: experiences and challenges", IJEPS, Vol. 13, pp. 3-26, 2019.

[2] Van der Veen, R. A. C., Hakvoort, R. A., "Balance responsibility and imbalance settlement in Northern Europe - An evaluation", 6th International Conference on the European Energy Market, Leuven, pp. 1-6, May 2009.

[3] Van der Veen, R. A. C., Hakvoort, R. A., "Balance responsibility and imbalance settlement in Northern Europe - An evaluation", 6th International Conference on the European Energy Market, Leuven, pp. 1-6, May 2009.

[4] Bianco, V., Manca, O., Nardini, S., "Electricity consumption forecasting in Italy using linear regression models", Energy, Vol. 34, No. 9, pp. 1413-1421, September 2009.

[5] Abdel-Aal, R.E., Al-Garni, A.Z., "Forecasting monthly electric energy consumption in eastern Saudi Arabia using univariate time-series analysis", Energy, Vol. 22, No. 11, pp. 1059-1069, November 1997.

[6] Akdi, Y., Gölveren, E., Okkaoğlu, Y., "Daily electrical energy consumption: Periodicity, harmonic regression method and forecasting", Energy, Vol. 191, January 2020.

[7] Gori, F., Takanen, C., Forecast of energy consumption of industry and household \& services in Italy", International Journal of Heat and Technology, Vol. 22, No. 2, pp. 115-121, January 2004.

[8] Azadeh, A., Ghaderi, S.F., Sohrabkhani, S., "Annual electricity consumption forecasting by neural network in high energy consuming industrial sectors", Energy Conversion and Management, Vol. 49, No. 8, pp. 22722278, August 2008

[9] Amber, K.P., Aslam, M.W., Hussain, S.K., "Electricity consumption forecasting models for administration buildings of the UK higher education sector", Energy and Buildings, Vol. 90, pp. 127-136, March 2015.

[10] http://www.uge.rs/

[11] Bratislav Predić, Nevena Radosavljević, Aleksandar Stojčić, "Time Series Analysis: Forecasting Sales Periods in Wholesale Systems", Facta Universitatis, Series: Automatic Control and Robotics, Vol. 18, No. 3, 2019, pp. $177-188$.

[12] Milojković, J.B., Litovski, V.B., "Short term forecasting in Electronics", International Journal of Electronics, Vol. 98, No. 2, pp. 161-172, February 2011.

[13] Igor Jovanović, Andrija Petrušić, Miona Andrejević Stošović, Dragan Mančić, "SARIMA and ANN approaches in day-ahead power consumption forecasting", Proceedings of 8th Small Systems Simulation Symposium, Faculty of Electronic Engineering, Niš, pp. 123-128, ISBN 978-86-6125-220-4, February 2020.

[14] A.L. Katsatos, K.P. Moustris, "Application of Artificial Neuron Networks as Energy Consumption Forecasting Tool in the Building of Regulatory Authority of Energy," Athens, Greece, Energy Procedia, Vol. 157, 2019, pp. 851-861, ISSN 1876-6102, https://doi.org/10.1016/j.egypro.2018.11.251.

[15] Adhiswara, R, Abdullah, A. G., Mulyadi, Y.,"Long-term electrical consumption forecasting using Artificial Neural Network", Journal of Physics: Conference Series, Vol. 1402, Issue 3, 2019.

[16] https://machinelearningmastery.com/adam-optimization-algorithm-for-deep-learning/

[17] https://www.tensorflow.org/ 